\title{
Identification of the ligand of Pru p 3, a peach LTP
}

\author{
Nuria Cubells-Baeza - Cristina Gómez-Casado - Leticia Tordesillas - Carmen Ramírez-Castillejo · \\ María Garrido-Arandia · Pablo González-Melendi · María Herrero · Luis F. Pacios · Araceli Díaz-Perales
}

\begin{abstract}
Key message Pru p 3, a peach LTP, is located in pollinated flower styles and secreting downy hairs, transporting a derivative of camptothecin bound to phytosphingosine. Pru p 3 may inhibit a second pollination and may keep away herbivores until seed maturation.

Abstract The allergen Pru p 3, a peach lipid transter protein, has been well studied. However, its physiological function remains to be elucidated. Our results showed that Pru p 3 usually carries a lipid ligand that play an essential role in its function in plants. Using ESI-qToF, we observed that the ligand was a derivative of camptothecin binding to phytosphingosine, wich that is inserted into the hydrophobic tunnel of the protein. In addition, the described ligand displayed topoisomerase I activity inhibition and self-fluorescence, both recognized as camptothecin properties. During flower development, the highest expression of Pru $p 3$ was detected in the styles of pollinated flowers, in contrast to its non-expression in unpollinated pistils, where expression decreased after anthesis. During ripening, the
\end{abstract}

expression of Pru p 3 were observed mainly in peel but not in pulp. In this sense, Pru p 3 protein was also localized in trichomes covering the fruit epidermis.

\section{Introduction}

Plant non-specific lipid transfer proteins (nsLTPs) are basic proteins with a molecular weight of about $9 \mathrm{kDa}$ (92-94 residues) and amino acid sequence identities ranging from 25 to 95\% (Kader 1996; Salcedo et al. 2007; Thoma et al. 1993). Despite the considerable heterogeneity in their primary structure, all LTPs share a compact $\alpha$-helical fold composed of four $\alpha$-helices connected by short loops and a non-structured $\mathrm{C}$-terminal tail. This fold is stabilized by eight conserved cysteine residues that form four disulfide bonds (Pacios et al. 2012; Salcedo et al. 2007; Salminen et al. 2016). A characteristic tunnel-like cavity aligned with the long axis of the protein is formed by the space left within the helices. Hydrophobic side chains coat the inner surface of this cavity, which is large enough to accommodate ligands with long chains (Hamilton 2004; Sossountzov et al. 1991; Zachowski et al. 1998). These properties point to a potential role for LTPS in membrane biogenesis, and some members are thought to have a role in defence against pathogens (Molina and Garcia-Olmedo 1993), transport of cutin monomers, and fruit and flower development (Chae et al. 2010; Salcedo et al. 2007; Salminen et al. 2016). The ability of plant nsLTPs to bind a wide variety of lipids enables them to perform various functions (Pacios et al. 2012). 
However, given that data on the association between compounds transported by LTPs and their specific functions are scarce in the literature, we expected that time- and tissue-specific expression of LTP genes would shed light on their physiological role in relation to the molecules they carry. Two LTPs, LTP1 (AY792996.1) and LTP2 (AY093699), are expressed in peach (Prunus persica) (Bonghi et al. 2011; Botton et al. 2009, 2002). Although LTP1 (Pru p 3 [IPR000528]) is expressed in pollinated flowers, it is poorly expressed in the ovary, and its expression is independent of pollination. LTP2 was highly expressed only in the ovary. By contrast, in fruit, only LTP1 mRNA was detected in the peel (Borges et al. 2006; Botton et al. 2009). Nevertheless, little is known about the physiological role of these LTPs and the specific molecules that they harbour and transport.

Alkaloids are a diverse group of low-molecular-weight, nitrogen-containing molecules found in about $20 \%$ of plant species. Many of the 16,000 alkaloids, for which structures have been described, participate in the defence of plants against herbivores, microbes, viruses, and competing plants (Wink 2003). Camptothecin is a cytotoxic quinoline alkaloid that was first isolated from the bark and stem of Camptotheca acuminata (Happy tree), a tree that is native to China and is used in traditional Chinese medicine. The biosynthesis of this alkaloid in almost all tissues has been described in many species (Lorence and Nessler 2004). Camptothecin is synthesized through a modified terpenoid indole alkaloid pathway in which there is a key enzyme: strictosidine synthase (STR), which is involved in the final step in the formation of the strictosidine backbone (Lorence and Nessler 2004). Gene expression involved in STR synthesis was found in leaves (more active in the young one), flowers (specially in petioles) and fruit and in most plant tissues (Isah and Mujib 2015; Sun et al. 2011; WenZhe 2004; Zhao et al. 2010). It is produced in pre-vacuol compartiments before being secreted through glandular trichomes. This mechanism has also been described for other toxic alkaloids such as those in Cannabis sativa (Sirikantaramas et al. 2005) and peppermint (Dudareva et al. 2004). Although not yet described in peach, the biosynthesis pathway of camptothecin was predicted from the peach genome (http://pathways.rosaceae.org), even though evidence for expression of the final steps of the pathway was lacking.

In this manuscript, we have characterized the ligand of Pru p 3 as a derivative of camptothecin bound to a long hydrophobic tail identified as phytosphingosine. By monitoring the expression of the Pru $p 3$ gene and genes of key enzymes in the biosynthesis pathway of both camptothecin and phytosphyngosine at different stages of flower and fruit development, we found that the ligand of Pru $\mathrm{p} 3$ may play a role in those processes. We propose that the Pru p 3-ligand complex could help prevent double pollination and defend against herbivores until the embryo (seed) has fully matured.

\section{Materials and methods}

\section{Isolation of Pru p 3}

Pru p 3 was purified as previously described (Diaz-Perales et al. 2002). Briefly, peach peel was separated by cation exchange chromatography on a SepPakR AccellPlus CM cartridge (Waters Corp, Milford, MA, USA) with $20 \mathrm{mM}$ formic acid, $\mathrm{pH}$ 4.0. The cationic retained fraction was then eluted with $0.75 \mathrm{mM} \mathrm{NaCl}$ in the same buffer $(1 \mathrm{~mL} /$ $\mathrm{min})$. This fraction was further purified by reversed-phase high-performance liquid chromatography (RP-HPLC) on a Nucleosil $300-\mathrm{C} 4$ column $(7 \times 250 \mathrm{~mm}$; particle size $5 \mu \mathrm{m}$ : Tecknokroma, Barcelona, Spain) to obtain the purified HPLC fraction. The fraction was eluted with a linear gradient of acetonitrile in $0.1 \%$ trifluoroacetic acid $(10 \%$ for $5 \mathrm{~min}$ and $10-100 \%$ over $150 \mathrm{~min} ; 1 \mathrm{~mL} / \mathrm{min}$ ).

Protein fractions were quantified using the bicinchoninic acid assay (Pierce, Cheshire, UK), and purity was measured using SDS-PAGE. Replicates were electrotransferred to PVDF membranes, and incubated with Pru p 3 polyclonal antibodies after blocking. The binding was revealed by HRP-chemiluminescence.

$\mathrm{N}$-terminal amino acid sequencing was performed with an Applied Biosystems 477A gas-phase sequencer (Applied Biosystems), and mass spectrophotometric analysis was performed with a Biflex III Spectrometer (Bruker-Franzen Analytik, Bremen, Germany). Fingerprinting analysis was performed using standard methods after trypsin digestion.

\section{Circular dichroism analysis}

The samples (cationic retained and HPLC purified fraction) were desalted using MicroSpin G25 Columns (GE Healthcare, United Kingdom). Circular dichroism measurements were performed in water on a J-810 spectropolarimeter (Jasco, Canada) using 1-mm path-length quartz cells equilibrated at $20^{\circ} \mathrm{C}$. Spectra were recorded from 190 to $260 \mathrm{~nm}$ with $0.5 \mathrm{~nm}$ resolution at a scan speed of $50 \mathrm{~nm} / \mathrm{min}$. Final spectra were baseline-corrected by subtracting the corresponding solvent spectra obtained under identical conditions. Data were fitted using the DichroWeb analytical tool. Results were expressed as the mean residue ellipticity $(\Theta)$ for a given wavelength.

\section{Isolation of the ligand of Pru p 3}

The ligand was separated from the retained cationic fraction by size-exclusion LH-20 chromatography using 
methanol:water (70:30) as the mobile phase. Fractions were checked using thin layer chromatography on silica gel-coated plates (Merck, USA) and developed with methanol:water $(70: 30 \mathrm{v} / \mathrm{v})$ in a saturated chromatography chamber for about $20 \mathrm{~min}$. The plates were visualized under UV light (254 $\mathrm{nm}$ ) and stained with vanillin ( $1 \%$ in ethanol) and dichlorofluorescein $(0.1 \%$ in ethanol- $\mathrm{NaOH}$ $2.5 \mathrm{mM}$ ) (Sigma-Aldrich, Germany). Fluorescent fractions were pooled and re-purified by HPLC as previously described (Kulkarni et al. 2010), with slight modifications. Isocratic analytical HPLC was performed using an RP-C18 column (Nucleosil $120 \mathrm{C} 18,5 \mu \mathrm{m}, 250 \times 4 \mathrm{~mm}$, Scharlab, Spain). The mobile phase for alkaloid elution was acetonitrile: water $(85: 15$ ) at a flow rate $0.5 \mathrm{~mL} / \mathrm{min}$ for $150 \mu \mathrm{L}$ of sample. Sample peaks were detected using UV light at $254 \mathrm{~nm}$. All reagents were HPLC grade.

\section{Pru p 3-ligand-binding assays}

To study the binding affinity of Pru p 3 for its ligand, increasing amounts of the ligand $(0.0001-1 \mu \mathrm{g})$ were dotted onto a nitrocellulose membrane. After blocking, the membrane was incubated with a solution of Pru p $3(1 \mu \mathrm{g} / \mu \mathrm{L})$, and binding was revealed by immunodetection using specific antibodies. After extensive washing, dot intensity was calculated using a Bio-Rad Pharos FX ${ }^{\mathrm{TM}}$ Plus Molecular Image. Binding affinity was expressed as the dissociation constant $\mathrm{Kd}=[$ Pru p 3][Ligand]/[Complex], where [Complex] was measured as the Pru p 3-bound form using a pattern curve with known amounts of the protein. All tests were performed in three independent assays.

\section{Mass spectrometry analysis of the ligand of Pru p 3}

The ligand of Pru p 3 was analysed using the HPLC system. Samples were separated by HPLC (Nucleosil 120 $\mathrm{Cl} 8,5 \mu \mathrm{m}, 250 \times 4 \mathrm{~mm}$, Tecknokroma) with an acetonitrile gradient of $10-85 \%$ for $30 \mathrm{~min}$ at $0.5 \mathrm{~mL} / \mathrm{min}$. Spectra were recorded at 254 and $280 \mathrm{~nm}$. Commercial camptothecin (Sigma, SL, USA), OH-camptothecin (Santa Cruz, Te, USA) and quercetin (Sigma, SL, USA) were used as standards.

Ionic fragmentation of the ligand of Pru p 3 was performed by diluting the ligand in acetonitrile containing $0.1 \%$ formic acid (v/v), and the solution was applied directly to the MALDI target. The analysis was performed in a micrOTOF-Q II mass spectrometer (Bruker Daltonik, Bremen, Germany) equipped with an ESI source in positive ion mode. Typical instrument settings were as follows: capillary voltage, $4500 \mathrm{~V}$; capillary exit, $130 \mathrm{~V}$; dry gas temperature, $180^{\circ} \mathrm{C}$; dry gas flow, $4 \mathrm{~L} / \mathrm{min}$. Spectra were obtained in positive ion mode. The mass spectrometer was used to carry out two scans: a full-mass scan between 50 and $1000 \mathrm{~m} / \mathrm{z}$ at a repetition rate of $2 \mathrm{~Hz}$, and an MS-MS scan of the most abundant ions in the full-mass scan. Argon was used as the collision gas. Collision energy was ramped at between 5 and $25 \mathrm{eV}$. Mass calibration was performed using sodium formate clusters (10-mM solution of $\mathrm{NaOH}$ in $50 / 50 \% \mathrm{v} / \mathrm{v}$ isopropanol/water containing $0.2 \%$ formic acid).

\section{Inhibition assays of topoisomerase I activity}

Since camptothecin is known to inhibit topoisomerase I, this activity was examined in the ligand of Pru p 3 using camptothecin assays, as reported elsewhere (Wright et al. 2015). We incubated $200 \mathrm{ng}$ of plasmid pBSK with $1 \mathrm{U}$ of human topoisomerase I (Inspiralis, UK) and increasing amounts of the ligand of Pru $\mathrm{p} 3$ after heating $\left(5 \mathrm{~min} 90^{\circ} \mathrm{C}\right.$ ) and $(S)$-(+)-camptothecin (Sigma-Aldrich, Germany) for $30 \mathrm{~min}$ at $37^{\circ} \mathrm{C}$. The reaction was run on a $0.8 \%$ agarose gel at $5-10 \mathrm{~V} / \mathrm{cm}$. The gel was then stained with ethidium bromide for $30 \mathrm{~min}$, washed briefly with water, and photographed in a UV transilluminator.

\section{Modelling of structures of the ligand and the Pru p 3-ligand complex}

The initial geometry of camptothecin was taken from the X-ray structure of its complex with human DNA topoisomerase I [PDB code 1T8I; (Wright et al. 2015)]. The initial geometry with the proper ( $2 \mathrm{~S}, 3 \mathrm{~S}, 4 \mathrm{R})$ configuration of phytosphingosine was firstly built up with modelling software Chimera 1.10.2 (Pettersen et al. 2004). The ligand model was constructed by binding the two molecules through an amide moiety formed by lactone carbonyl from camptothecin and amine nitrogen from phytosphingosine. Hydroxyl was added to camptothecin $\mathrm{C} 10$, and the whole geometry was optimized. Hydrogens were then added and several geometry minimization cycles were run. This modelling was also performed with Chimera 1.10.2 (Pettersen et al. 2004).

Phytosphingosine alone was docked in the hydrophobic cavity of the crystal structure of Pru p 3 PDB code 2ALG; (Pasquato et al. 2006) by means of AutoDock Vina calculations (Trott and Olson 2010). Three docking results with the lowest affinity energies were used to prepare three initial geometries of the Pru p 3-ligand complex that then underwent 10-ns molecular dynamics (MD) simulations in water to find optimized structures. These calculations were performed with NAMD 1.10 (Phillips et al. 2005) according to a recently reported procedure (Garrido-Arandia et al. 2014). Force field parameters for the modelled ligand were assigned using SwissParam (ZoetteV et al. 2011). One of the three structures showed the best results for stability of ligand atoms (lowest RMSD), protein-ligand interaction 
energy, and total energy of the complex upon completion of the three MD simulations. This optimum structure was the final model for the Pru p 3-ligand complex (see below).

\section{Plant material}

Tissues from the Super Crimson Gold peach cultivar were used to study gene expression during flower development and fruit set. Pollinated and unpollinated flowers at different time points $(-2,-1,0,1,7,14,21,26,28,35,51,58$, $65,73,80,87,94,101$ and 105 days post-anthesis [dpa]) from anthesis to mature fruit. The anthers, stigma-style, ovary, and pedicel from the flower and seed and peel from the fruit were excised separately, frozen immediately in liquid nitrogen, and stored at $-80^{\circ} \mathrm{C}$ for RNA study.

Pollen was recovered from the same flowers by shaking and stored immediately at $-80^{\circ} \mathrm{C}$. For testing pollen germination, grains were incubated in $15 \%$ glucose in darkness. Pru p $3(1 \mu \mathrm{g} / \mathrm{mL})$ and the Pru p 3-ligand complex $(0.1 \mu \mathrm{g} / \mathrm{mL})$ were added to the solution directly.

\section{Real-time PCR in flower and fruit tissues}

Total RNA was extracted from the tissues following the method published (Tordesillas et al. 2013). Gel electrophoresis $(1 \%$ agarose) and spectrophotometry $(260 / 280 \mathrm{~nm})$ were used to determine RNA quality and concentration. The first-strand cDNA was synthesized from $1 \mu \mathrm{g}$ of DNAfree total RNA treated with DNase I (Roche, Basle, Switzerland) using random primers and a High Capacity cDNA Reverse Transcription kit (Applied Biosystems, CA, USA).

Relative real-time PCR quantification was performed using Power SYBR Green PCR Master Mix (Applied Biosystems) according to the manufacturer's recommendations. Primers $18 \mathrm{~S}-\mathrm{F}$ and $18 \mathrm{~S}-\mathrm{R}$ (5'-GGGCATTCGTAT TTCATAGTCAGAG-3' and 5'-GGTTGAGACTAGGAC GGTA TCTGA-3') were used to amplify a $146-b p$ fragment of the endogenous control $18 \mathrm{~S}$ from Prunus persica (GenBank Accession No. L28749).

Primers Pru p 3-F and Pru p 3-R (5'-TGTGCATGG TTGTGAGCGTG- $3^{t}$ and $5^{t}$-GTTGTTGACGTTCCTAAT GCCG-3') were designed to amplify a 143-bp fragment of Pru p 3 gene from P. persica (GenBank Accession No. AY792996).

The thermal cycling conditions were as follows: $10 \mathrm{~min}$ at $95^{\circ} \mathrm{C}, 15 \mathrm{~s}$ at $95^{\circ} \mathrm{C}$, and $45 \mathrm{~s}$ at $60^{\circ} \mathrm{C}(40$ cycles). The optimal cDNA and primer concentrations were determined by serial dilution experiments. Finally, $10 \mathrm{ng}$ of cDNA per well were added, and the final concentration of the primers was $1.5 \mu \mathrm{M}$. The amount of Pru p 3, STR, and $S 4 D$ mRNA expression was normalized using the endogenous control 18S, and relative quantification was performed using the comparative threshold cycle method $(2-\Delta \Delta \mathrm{Ct})$, as previously described (Tordesillas et al. 2013). Changes in gene expression were calculated with reference to anthesis $(7 \mathrm{dpa})$. The products of reactions were separated in $2 \%$ agarose gel and stained with ethidium bromide. All PCR experiments were carried out in triplicate, in three independent experiments.

\section{Immunofluorescence and confocal microscopy}

To detect the presence of Pru p 3, the samples were fixed with $4 \%$ formaldehyde in PBS (pH 7.4) at $4^{\circ} \mathrm{C}$ overnight. After washing in PBS and sectioning with a razor blade, the tissues were permeabilized ( 20 freezing/thawing cycles of 5 min each). Then, the specimens were washed with PBS and blocked with 5\% BSA in PBS for $1 \mathrm{~h}$. Immunohistochemistry was performed in whole-mount tissue by incubating overnight at $4^{\circ} \mathrm{C}$ with an anti-Pru p 3 polyclonal antibody [applied 1:50 in PBS; (Palacin et al. 2012)] and a secondary antibody, Alexa 488-conjugated anti-rabbit antibody (applied 1:500 in PBS, Molecular Probes). The tissue sections were mounted with glycerol:PBS (1:1) on 10-well slides (FisherScientific Inc., Pittsburgh, USA) and observed with a Leica TCS-SP8 confocal microscope using the laser excitation lines of $488 \mathrm{~nm}$ for the localization of Pru p 3 and $405 \mathrm{~nm}$ to detect alkaloid autofluorescence. Measurements of fluorescence intensity in ten independent ROIs on $\mathrm{Z}$ stack maximal projections were used to compare the immuno-expression of Pru p 3 protein and the alkaloid.

\section{Statistical analyses}

Statistical analyses were performed using SPSS 17.0 and Statgraphics Centurion XVI. Gene expression was compared using the Mann-Whitney test. Statistical significance was set at $\mathrm{p}<0.05$ for all analyses. At least three independent experiments were used for all assays.

\section{Results}

\section{Pru p 3 carried a lipid compound}

Pru p 3 was isolated from peach peel following two chromatographic steps: cationic exchange (cationic-retained) and reversed phase (HPLC purified), as previously described (Diaz-Perales et al. 2000). The presence of a lipid compound bound to Pru p 3 was confirmed by thin layer chromatography in methanol as the mobile phase and stained with vanillin (Fig. 1b). A lipid stain was observed in the cationic-retained fraction, whereas no spot was found in the HPLC-purified protein.

The secondary structure of Pru p 3 from the cationicretained fraction was studied using circular dichroism and 
(A)

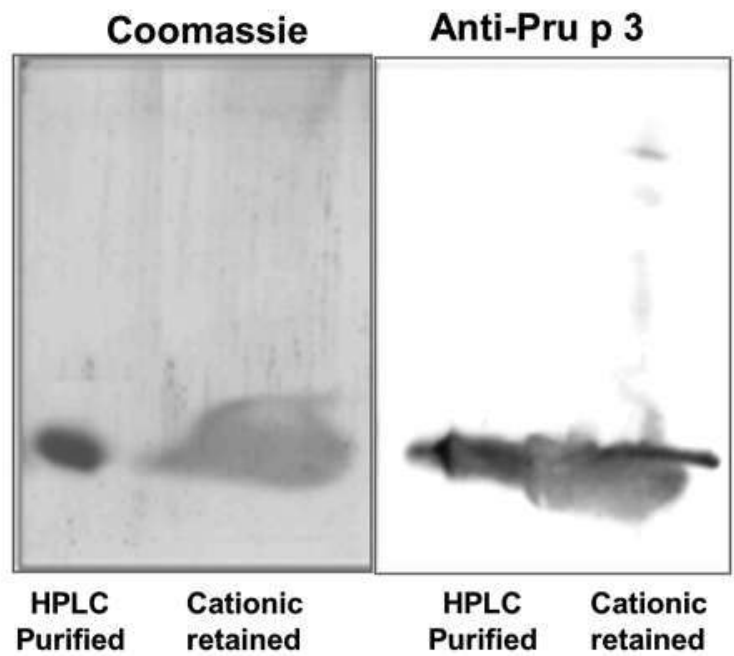

(C)

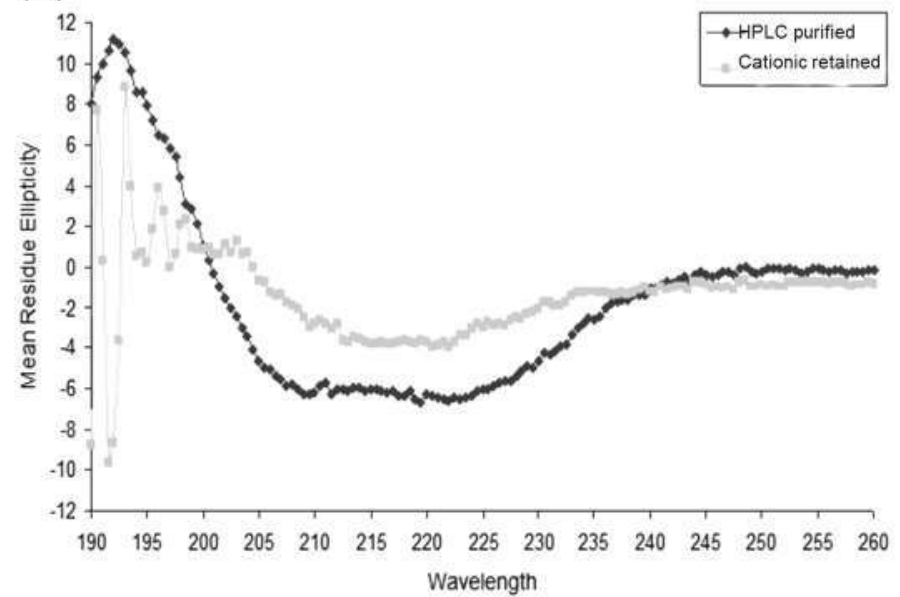

(B) Vanillin

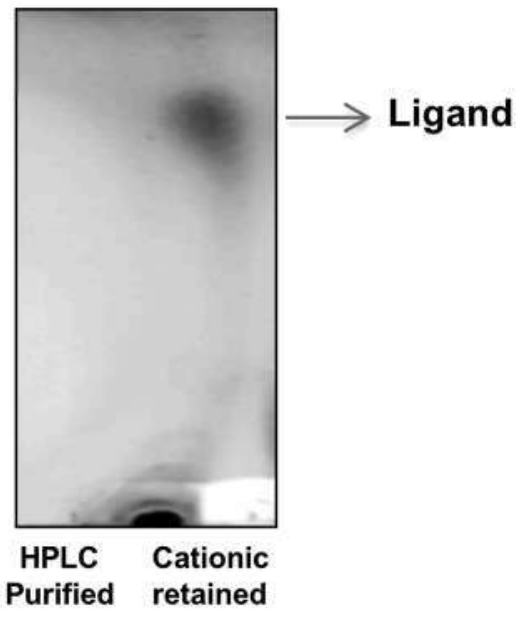

(D)



Fig. 1 a The retained fraction from cationic chromatography $(5 \mu \mathrm{g}$; Cationic retained $)$ and purified Pru p $3(5 \mu \mathrm{g} ;$ HPLC purified $)$ were separated by SDS-PAGE and stained with Coomassie R-250 (Coomassie) or incubated with polyclonal antibodies (anti-Pru p 3). b Both fractions were also separated in silica gel-coated plates (Merck) using thin layer chromatography (TLC) in methanol:water (7:3 v/v) in a saturated chromatography chamber for about $20 \mathrm{~min}$. The plates were stained with vanillin (Sigma-Aldrich, Steinheim, Germany). c Both fractions were used to compare their secondary structure by far-

revealed an alteration in the far UV region, a feature that is typical of lipid binding (Fig. 1c). In contrast, HPLC-purified protein from RP-HPLC showed a circular dichroism spectrum typical of helices with no alteration in this region.

To characterize the interaction between Pru p 3 and this lipid compound, i.e. the ligand of peach LTP, the compound was isolated from the cationic-retained fraction by exclusion chromatography ( $70 \%$ methanol), followed by RP-HPLC in acetonitrile. The lipid fraction was
UV (190-260 nm) circular dichroism spectrum in water on a JASCO Spectropolarimeter J-810 (Jasco, Victoria, Canada) using 1-mm pathlength quartz cells equilibrated at $20^{\circ} \mathrm{C}$. Spectra were recorded from 190 to $260 \mathrm{~nm}$ with $0.5 \mathrm{~nm}$ resolution at a scan speed of $50 \mathrm{~nm} / \mathrm{min}$. Data were fitted using the Dichroweb analytical tool. d Increasing amounts of the ligand of Pru p 3 was dot-blotted were incubated onto a nitrocellulose membrane. After blocking, it was incubated with a Pru p 3 solution $(1 \mu \mathrm{g} / \mathrm{mL})$ and the binding was revealed by specific antibodies

confirmed as the ligand of Pru p 3 by dot-blot binding assay. Increasing amounts of this fraction diluted in PBS were dotted onto a nitrocellulose membrane, and, after blocking, the membrane was incubated with Pru p 3 solution $(1 \mu \mathrm{g} / \mu \mathrm{l})$. The interaction was revealed by specific antibodies (Fig. 1d). These results showed tight binding affinity between Pru p 3 and its ligand, represented by a dissociation constant estimated at $\mathrm{Kd}=0.2 \mu \mathrm{M}$ in a $1: 1$ ratio. 


\section{The ligand of Pru p 3 included a hydroxylated derivative of camptothecin bound to phytosphingosine}

The identification of the ligand was analysed using standard compounds and by comparing retention times in HPLC (Fig. 2a). The ligand showed a peak overlapping to $\mathrm{OH}$-camptothecin.

The presence of hydroxy-camptothecin and phytosphingosine were identified by ion fragmentation (Fig. 2b). Based on the pattern described for ceramides (Shin et al. 2014) and camptothecin (Zhao 2010), a fragmentation for the ligand of Pru p 3 was studied. The characteristic camptothecin fragment ions were detected as $364.81 \mathrm{~m} / \mathrm{z}$ and $202.83 \mathrm{~m} / \mathrm{z}$. Phytosphingosine fragment ions were identified as $218.79 \mathrm{~m} / \mathrm{z}$ and $230.01 \mathrm{~m} / \mathrm{z}$. The results also revealed peaks corresponding to both bound compounds, as $380.77 \mathrm{~m} / \mathrm{z}$ and $424.82 \mathrm{~m} / \mathrm{z}$.

The chemical identification of the ligand of Pru $p$ 3 was also confirmed by its capacity to emit blue light when excited with UV radiation (254 nm; Fig. 3a), a feature also shown by camptothecin (Sirikantaramas et al. 2007). Moreover, camptothecin is well known for its ability to inhibit the catalytic activity of topoisomerase I, the enzyme responsible for relaxing supercoiling DNA (Wright et al. 2015). Activity assays performed with the ligand of Pru p 3 clearly showed inhibition of topoisomerase I after heating, whereas no inhibition was observed when the ligand was used directly (data not shown) (Fig. 3b). The inhibitory activity of topoisomerase I occurs through the closed E-ring of camptothecin (Fig. 4a). However, linking to phytosphingosine through the amide bond in the ligand of Pru p 3, forces the open form of the E-ring, thus rendering the ligand inactive in the inhibition of topoisomerase. Given that this $\alpha$-hydroxy-lactone E-ring is susceptible to hydrolysis upon thermal treatment, the inhibition results are consistent with the chemical nature proposed for the ligand of Prup 3.
Fig. 2 a The ligand of Pru p 3 was separated by phase reverse-HPLC. Commercial OH-Camptothecin/, camptothecin and quercetin were used as standards. b ESI-micrOTOF-Q II. Deconvolution chromatogram corresponding to the camptothecin obtained from the ligand fraction when it was dissolved in methanol-formic acid

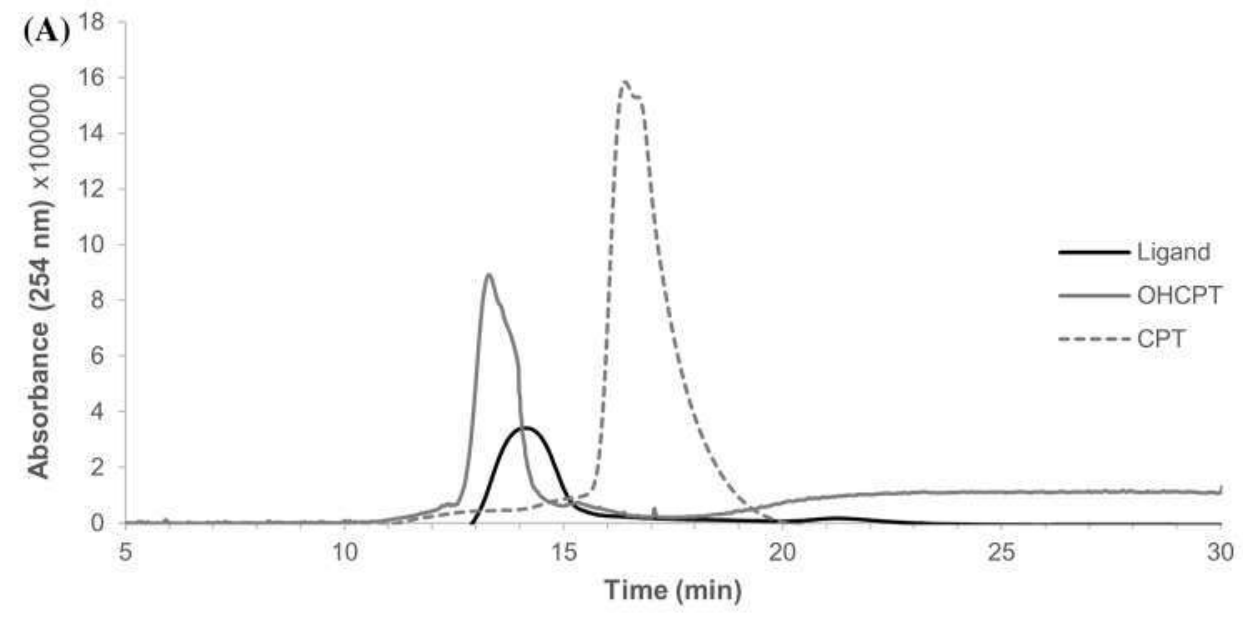

(B)




(A)
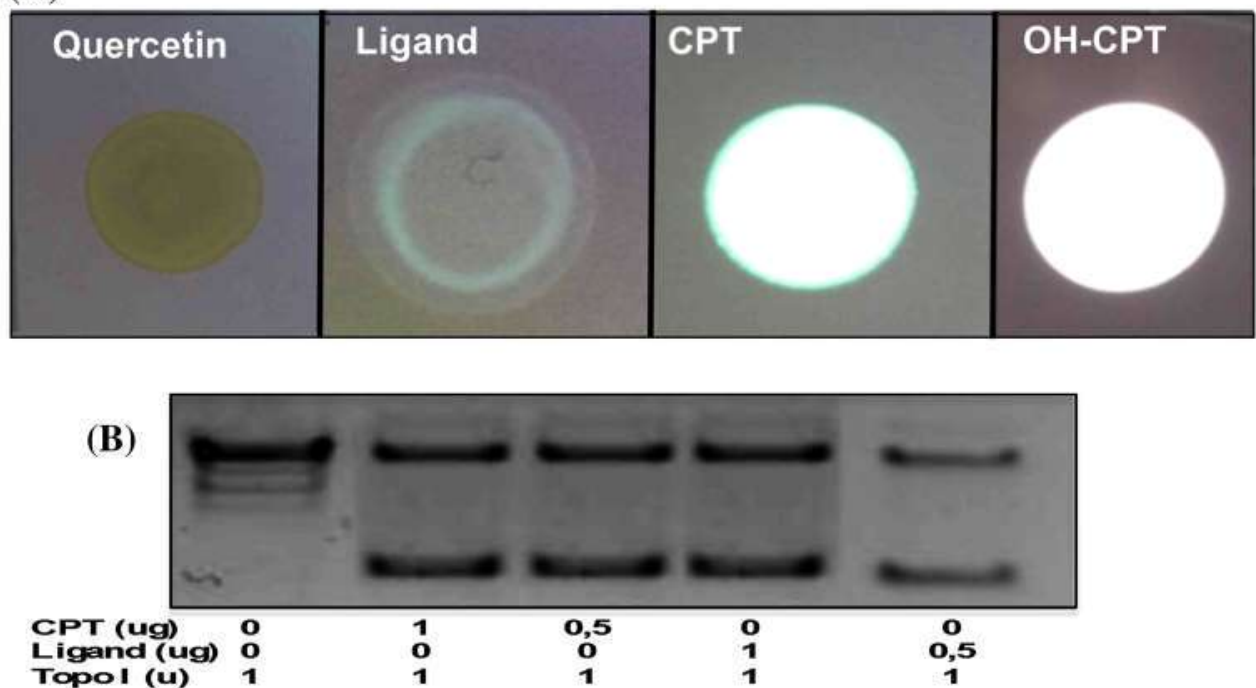

Fig. 3 a Commercial quercetin ( $1 \mu \mathrm{g}$ Sigma), the ligand of Pru p 3 (1 $\mu \mathrm{g}$; Ligand), commercial camptothecin (1 $\mu \mathrm{g}$ Sigma; CPT) and hydroxy-camptothecin ( $1 \mu \mathrm{g}$ Santa Cruz; OH-CPT) were loaded onto a silica gel plates and UV light (354 nm) was applied. b Inhibition of topoisomerase I activity. Plasmid pBSK (200 ng) was incubated with topoisomerase I ( $1 \mathrm{u}$; Inspiralis, UK) for $30 \mathrm{~min}$ at $37^{\circ} \mathrm{C}$ in the presence of camptothecin ( $1 \mu \mathrm{g}, 0.5 \mu \mathrm{g}$; Sigma, UK) or of the ligand $(1 \mu \mathrm{g}, 0.5 \mu \mathrm{g})$, heated for $5 \mathrm{~min}$ at $90^{\circ} \mathrm{C}$. After the mix was separated on a $0.8 \%$ agarose gel at $5-10 \mathrm{~V} / \mathrm{cm}$, the gel was stained with ethidium bromide $(10 \mathrm{mg} / \mathrm{mL})$ and distained briefly with water. The gel was illuminated with a UV transilluminator

et al. 2012) at a remarkably similar position (Fig. 4d). Given that this spatial coincidence arises from superposition of proteins only, the finding lends strong support to our model structure.

\section{The Pru p 3 gene is expressed mainly in the style of pollinated flowers}

Expression of the Pru $p 3$ gene revealed maximum levels in the flower, particularly high in the stigma-style in contrast to that observed in the ovary and pedicel, where the expression was very low (about 1000 -fold less than in the stigma-style; Fig. 5a). In the style of non-pollinated flowers, Pru $p 3$ expression decreased after the flower opened and continued to decrease after that stage. However, in pollinated flowers, Pru p 3 expression increased from 0 dpa until 21 dpa.

These results were confirmed by immunofluorescence assays in whole-mount tissue (Fig. 6). Pru p 3 was located specifically in the pollinated flower stigma from 7 to 21 dpa, with a maximum expression at $21 \mathrm{dpa}$, which fell and then finally disappeared at $28 \mathrm{dpa}$.

\section{The Pru p 3-ligand complex inhibited pollen germination}

To gain a deeper understanding of the role of the Pru p 3-ligand complex in pollination, pollen grains were 
Fig. 4 a Structural formula of the modeled ligand of Pru p 3 composed of 10-hydroxy-camptothecin (red) and phytosphingosine (blue). b Model structure of the Pru p 3 (ribbon)-ligand (sticks with $\mathrm{C}$, green; $\mathrm{N}$, blue; and $\mathrm{O}$, red) complex. $\mathrm{c}$ Molecular surface of the Pru p 3-ligand complex structure shown in b. d Superposition of proteins in wheat LTP (light orange ribbon)-LMPC (sticks with carbons in yellow) complex (crystal structure, PDB id 1BWO) and Pru p 3 (green ribbon)-ligand (sticks with carbons in green) complex (model structure, present study)

germinated in $15 \%$ glucose for $4 \mathrm{~h}$ in the absence of Pru $\mathrm{p}$ 3 (Control) and in the presence of $1 \mu \mathrm{g} / \mathrm{mL}$ of Pru p 3 (Pru p 3$)$ or Pru p $3(1 \mu \mathrm{g} / \mathrm{mL})$ together with its ligand $(0.1 \mu \mathrm{g} /$ $\mathrm{mL}$ ) (Pru p $3+$ ligand). Germination was essentially inhibited in the presence of the Pru p 3-ligand complex (Fig. 5b).

\section{The Pru p 3 gene is expressed mainly in the trichomes of peel}

In the case of fruit development (Fig. 5c), expression of the Pru $p 3$ gene was concentrated in the peel, starting at 70 dpa and reaching its maximum at $80 \mathrm{dpa}$. From that time on, mRNA levels decreased to $105 \mathrm{dpa}$.

Thus, Pru p 3 protein was also located mainly in the peel of ripe fruits, particularly on trichomes (Fig. 7). Interestingly, the alkaloid signal was also localized on peach peel trichomes; this finding is consistent with the kinetics of expression of Pru $p 3$ obtained in our study.

\section{Discussion}

The present study is the first to characterize the ligand of the peach LTP Pru p 3 as a derivative of camptothecin bound to phytosphingosine. Ligand binding to LTPs is accompanied by slight changes in the unstructured C-terminal tail of the protein, which acts as a lid on the hydrophobic cavity, although the $\alpha$-helical fold is preserved (Salcedo et al. 2007; Salminen et al. 2016). Consistent with the behaviour described for LTPs upon ligand binding, the presence of the ligand produced a distortion in the circular dichroism spectrum of Pru p 3 in the vacuum UV region (190-200 nm).

In the mass spectrometry analysis, the ligand of Pru p 3 suggested the presence of two different chemical species, each of which was identified as a monohydroxylated derivative of camptothecin and phytosphingosine. The presence of camptothecin was also confirmed by its autofluorescence properties and its ability to inhibit topoisomerase I activity, two well-known features of this alkaloid. However, given their chemical structures, both molecules barely occupy the hydrophobic central cavity of Pru p 3. We therefore suggest that the two species forming the ligand and are linked through an amide bond that involves the
(A)

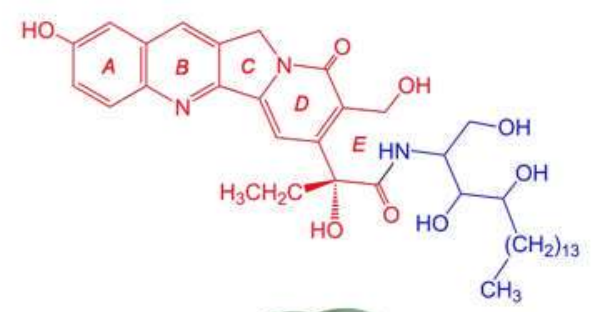

(B)



(C)
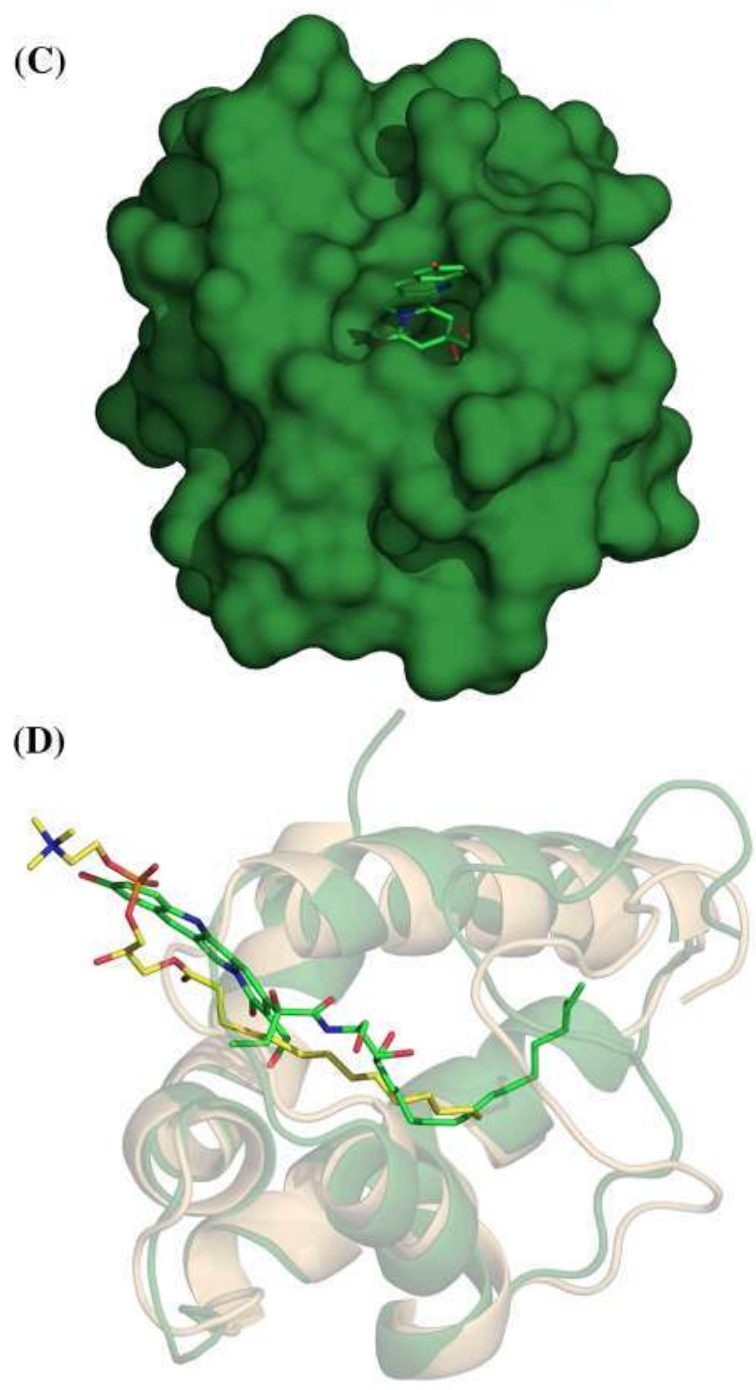


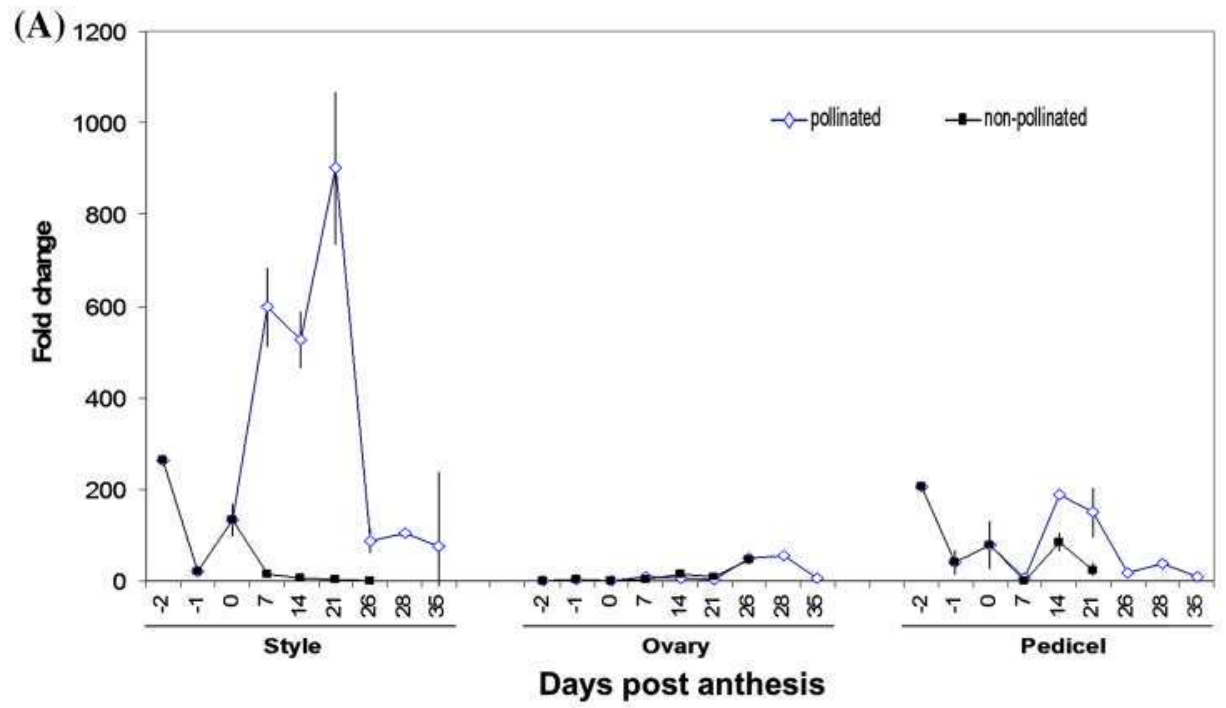

(B)

Control Pru p $3 \quad$ Pru p 3+ ligand $\%$ of germinated grains
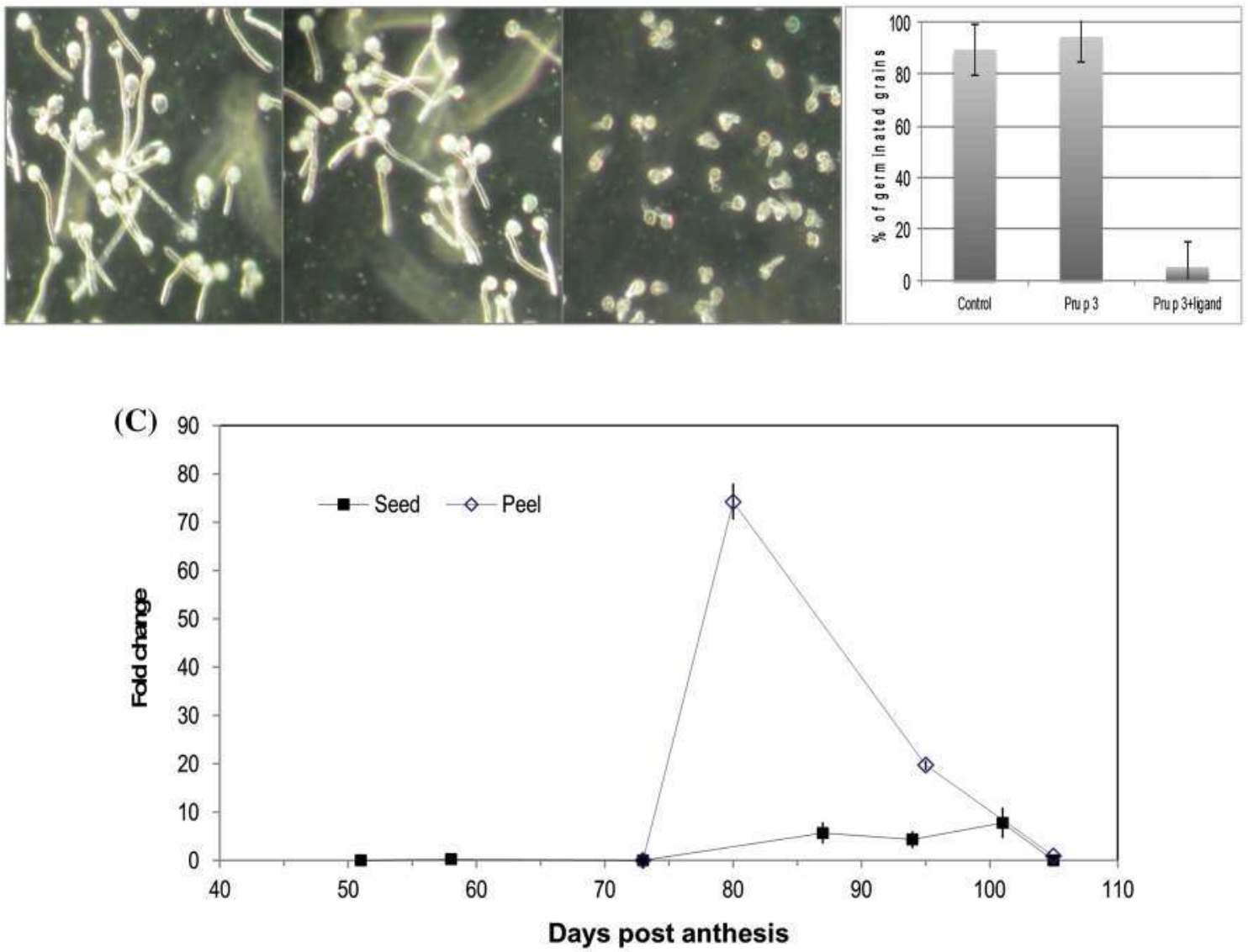

Fig. 5 a Expression profile of $P r u p 3$ gene in style, ovary, and pedicel of peach flower collected from -2 to 35 days post-anthesis (dpa), in pollinated and unpollinated flower. All expression values are normalized to the $18 \mathrm{~S}$ gene. Relative expression is calibrated on the corresponding gene expression at ovary ' 0 ' time. The bars show the standard error. $\mathbf{b}$ Effect of the ligand on the pollen germination pro- cess. Pollen grains were grown in $15 \%$ glucose for $4 \mathrm{~h}$, in the absence of Pru p 3 (Control), in the presence of Pru p $3(1 \mu \mathrm{g} / \mathrm{mL} ;+$ Pru p 3$)$, and in the presence of Pru p 3 and its ligand $(0.1 \mu \mathrm{g} / \mathrm{mL} ;+$ Pru p 3 and ligand). The percentage of germinated grains (\%) was quantified in both samples. $\mathrm{c}$ Expression profile of Pru p 3 gene in peach peel and seed fruit collected from 51 to $105 \mathrm{dpa}$ 


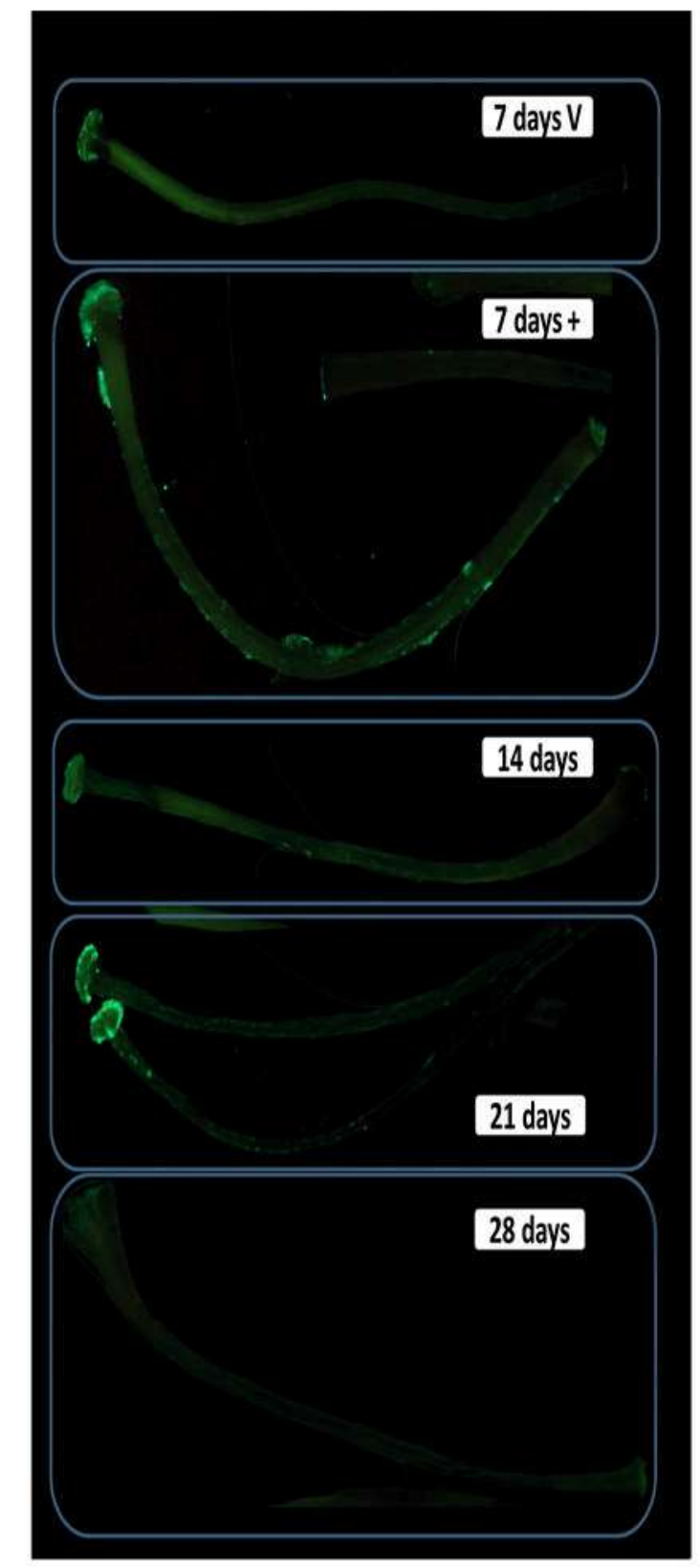

Fig. 6 Immunolocalization of Pru p 3 in styles from flowers collected at different dpa. v, unpollinated flowers; + , pollinated flowers. Pru p 3 was detected using a specific monoclonal antibody, which showed fluorescence signal in green (Pru p 3 revealed by Alexa 488)

E-ring of camptothecin, which is known to be responsible for the inhibitory activity of topoisomerase I in its closed form (Wright et al. 2015). Since sphingoid molecules are also known to bind disparate moieties as substituents of amino hydrogens, the amide bond seems a reasonable link between alkaloid and sphingoid components of the ligand.

This hypothesis is supported by the data obtained in the mass spectometry spectra in which the camptothecin ions with the amide bond are identified by the peaks

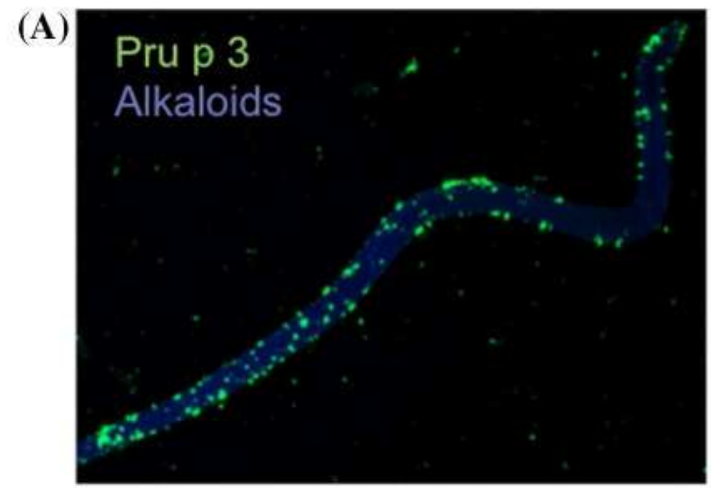

(B)



(C)



Fig. 7 Immunolocalization of Pru p 3 in trichomes from mature fruits ( $105 \mathrm{dpa})$ detected using specific monoclonal antibody. The fluorescence signal is shown in green (Alexa 488) and alkaloids in blue in $\mathbf{a}$; only blue light is observed in $\mathbf{b}$; and the face contrast in $\mathbf{c} . \times 40$

with $\mathrm{m} / \mathrm{z}$ values of 380.77 and 424.82 . The phytosphingosine segment is able to occupy the hydrophobic cavity of Pru p 3 because of the hydrophobic nature of amino acid side chains inside the cavity, which stabilizes binding of the hydrocarbonated nonpolar tail while leaving the polar groups close to the amide bound with camptothecin exposed to the solvent. Thus, given that the E-ring is open when the two segments are bound, the ligand of Pru p 3 lacks the pro-apoptotic activity characteristic of the alkaloid, and the LTP then merely transports an inactive compound causing no plant cell damage. However, if the ligand is released in the apoplast, hydrolysis of the E-ring breaks the bound to phytosphingosine, and camptothecin is 
released in the closed form of the ring, thus regaining its activity and inhibiting cell division and pollen tube growth. The link can be also broken by extreme conditions such as heat and acid treatment. Therefore, the analysis with high voltage lasers in mass spectrometers could be responsible for splitting both molecules, thus explaining the presence of the two peaks observed.

The fact that the phytosphingosine tail forces the E-ring of camptothecin to remain open could be interpreted as a detoxification mechanism. In many cases, secondary metabolites and their pathway intermediates are phytotoxic. Plants have evolved several defence mechanisms to avoid self-toxicity from secondary metabolites (Sirikantaramas et al. 2007). A basic strategy to avoid self-toxicity is tight regulation of biosynthesis in terms of temporal/spatial gene expression and localization. In this sense, the highest expression of Pru 3 and the Pru p 3-ligand activity in pollinated stigma-styles led us to propose that both features might inhibit double pollination. Moreover, the capacity to inhibit pollen germination, together with timing of expression, also suggests a role in preventing the pollination if one considers that maximum expression has been shown to match fertilization time in peach flowers (Herrero and Arbeloa 1989).

Similarly, both the timing of Pru p 3 expression and the presence of the Pru p 3-ligand complex in fruit peel (mainly in trichomes), might also inhibit predator feeding in fruit. The maximum expression of Pru $p 3$ coincides with the end of embryo maturation and, thus, with the end of lignin deposition (Dardick et al. 2010) and accumulation of storage substances (amino acids and sorbitol) (Lombardo et al. 2011). Protection associated with expression of defence proteins such as LTPs is therefore necessary at that time. LTPs are expressed mainly in the cells of the exocarp in unicellular trichomes secreting alkaloids, as evidenced by immunolocalization of Pru p 3 and ultraviolet fluorescence. Hence, Pru p 3 might prevent ingestion of fruit until the embryo is fully developed.

In summary, Pru $\mathrm{p} 3$ can carry a monohydroxylated derivative of camptothecin bound to phytosphyngosine. This LTP is expressed at two key times of flower and fruit development in peach, namely, during pollination and during embryo development. In both stages, the ligand of Pru p 3 can inhibit cell division, although with two different objectives: first, to avoid pollination, and second, to prevent the plant from herbivores.

Acknowledgements The authors thank the Centro de Supercomputación y Visualización de Madrid (CeSViMa) and Centre for Metabolomics and Bioanalysis (CEMBIO) for computing resources and technical assistance. The study was supported by the Ministry of Science and Inoovation (project BIO2013-041403-R) and FISThematic Networks and Co-operative Research Centres: RIRAAF (RD12/0013/0014). The funders had no role in the study design, data collection and analysis, the decision to publish, or the preparation of the manuscript.

Author contributions Sample collection: $\mathrm{MH}$ : Experimental design and analysis of results: PGM. MH, LFP, AD-P; Experimental development: NCB. CGC, LT; Modelling and molecular dynamics analysis: MGA, LFP; Confocal microscopy: CRC, PGM.

\section{Compliance with ethical standards}

Conflict of interest The authors declare that they have no competing interests.

\section{References}

Bonghi $C$ et al (2011) A microarray approach to identify genes involved in seed-pericarp cross-talk and development in peach. BMC Plant Biol 11:107

Borges JP. Jauneau A, Brule C. Culerrier R, Barre A. Didier A, Rouge $P(2006)$ The lipid transfer proteins (LTP) essentially concentrate in the skin of Rosaceae fruits as cell surface exposed allergens. Plant Physiol Biochem 44:535-542

Botton A. Begheldo M, Rasori A, Bonghi C. Tonutti P (2002) Differential expression of two lipid transfer protein genes in reproductive organs of peach (Promus persica L. Batsch). Plant Sci 163:993-1000

Botton A, Andreotti C, Costa G. Ramina A (2009) Peach (Prtnus persica $\mathrm{L}$. Batsch) allergen-encoding genes are developmentally regulated and affected by fruit load and light radiation. J Agricu Food Chem 57:724-734

Chae K, Gonong BJ. Kim SC. Kieslich CA. Morikis D. Balasubramanian S. Lord EM (2010) A multifaceted study of stigma/style cysteine-rich adhesin (SCA)-like Arabidopsis lipid transfer proteins (LTPs) suggests diversified roles for these LTPs in plant growth and reproduction. J Exp Bot 61:4277-4290

Charvolin D. Douliez JP. Marion D. Cohen-Addad C, Pebay-Peyroula E (1999) The crystal structure of a wheat nonspecific lipid transfer protein (ns-LTP1) complexed with two molecules of phospholipid at 2.1 A resolution. Eur J Biochem/FEBS 264:562-568

Dardick CD, Callahan AM, Chiozzotto R, Schaffer RJ, Piagnani MC, Scorza R (2010) Stone formation in peach fruit exhibits spatial coordination of the lignin and flavonoid pathways and similarity to Arabidopsis dehiscence. BMC Biol 8:13

Diaz-Perales A et al (2000) Lipid-transfer proteins as potential plant panallergens: cross-reactivity among proteins of Artemisia pollen. Castanea nut and Rosaceae fruits, with different IgE-binding capacities. Clin Exp Allergy 30:1403-1410

Diaz-Perales A, Garcia-Casado G. Sanchez-Monge R, Garcia-Selles FJ. Barber D. Salcedo G (2002) cDNA cloning and heterologous expression of the major allergens from peach and apple belonging to the lipid-transfer protein family. Clin Exp Allergy $32: 87-92$

Dudareva N. Pichersky E, Gershenzon J (2004) Biochemistry of plant volatiles. Plant Physiol 135:1893-1902

Garrido-Arandia M. Gomez-Casado C, Diaz-Perales A, LF P (2014) Molecular Dynamics of major allergens from Alternaria, birch pollen and peach. Mol Inf 33:682-694

Hamilton JA (2004) Fatty acid interactions with proteins: what X-ray crystal and NMR solution structures tell us. Prog Lipid Res 43:177-199

Herrero M. Arbeloa A (1989) Influence of the pistil on pollen tube kinetics in peach (Prttrus persica (L.) Batsch). Am J Bot 176:1441-1447 
Isah T, Mujib A (2015) Camptothecin from Nothapodytes nimmoniana: review on biotechnology applications. Acta Physiologiae Plantarum 37:1-14

Kader JC (1996) Lipid-transfer proteins in plants. Annu Rev Plant Physiol Plant Mol Biol 47:627-654

Kulkarni AV, Patwardhan AA, Lele U, Malpathak NP (2010) Production of camptothecin in cultures of Chonemorpha grandiflora. Pharmacognosy Res 2:296-299

Lombardo VA et al (2011) Metabolic profiling during peach fruit development and ripening reveals the metabolic networks that underpin each developmental stage. Plant Physiol 157:1696-1710

Lorence A, Nessler CL (2004) Camptothecin, over four decades of surprising findings. Phytochemistry $65: 2735-2749$

Molina A, Garcia-Olmedo F (1993) Developmental and pathogeninduced expression of three barley genes encoding lipid transfer proteins. Plant J 4:983-991

Pacios LF, Gomez-Casado C, Tordesillas L, Palacin A, SanchezMonge R, Diaz-Perales A (2012) Computational study of ligand binding in lipid transfer proteins: structures. interfaces. and free energies of protein-lipid complexes. J Comput Chem $33: 1831-1844$

Palacin A et al (2012) Graph based study of allergen cross-reactivity of plant lipid transfer proteins (LTPs) using microarray in a multicenter study. PloS One $7:$ e50799

Pasquato N. Berni R, Folli C. Folloni S, Cianci M. Pantano S. Helliwell JR. Zanotti G (2006) Crystal structure of peach Pru p 3. the prototypic member of the family of plant non-specific lipid transfer protein pan-allergens. J Mol Biol 356(3):684-694

Pettersen EF, Goddard TD, Huang CC, Couch GS, Greenblatt DM, Meng EC, Ferrin TE (2004) UCSF Chimera-A visualization system for exploratory research and analysis. J Comput Chem 25(13): 1605-1612

Phillips JC, Braun R, Wang W. Gumbart J, Tajkhorshid E. Villa E. Chipot C. Skeel RD. Kalé L, Schulten K (2005) Scalable molecular dynamics with NAMD. J Comput Chem 26(16):1781-1802

Salcedo G, Sanchez-Monge R, Barber D. Diaz-Perales A (2007) Plant non-specific lipid transfer proteins: an interface between plant defence and human allergy. Biochim Biophys Acta 1771:781-791

Salminen TA, Blomqvist K, Edqvist J (2016) Lipid transfer proteins: classification, nomenclature, structure, and function. Planta 244:971-997
Shin JH et al (2014) A lipidomic platform establishment for structural identification of skin ceramides with non-hydroxyacyl chains. Anal Bioanal Chem 406:1917-1932

Sirikantaramas S, Taura F, Tanaka Y, Ishikawa Y, Morimoto S, Shoyama Y (2005) Tetrahydrocannabinolic acid synthase, the enzyme controlling marijuana psychoactivity, is secreted into the storage cavity of the glandular trichomes. Plant Cell Physiol $46: 1578-1582$

Sirikantaramas S. Sudo H, Asano T, Yamazaki M, Saito K (2007) Transport of camptothecin in hairy roots of Ophiorrhiza pumila. Phytochemistry 68:2881-2886

Sossountzov L et al (1991) Spatial and temporal expression of a maize lipid transter protein gene. Plant Cell 3:923-933

Sun Y et al (2011) Pyrosequencing of the Camptotheca acuminata transcriptome reveals putative genes involved in camptothecin biosynthesis and transport. BMC Genom 12:533

Thoma S, Kaneko Y, Somerville C (1993) A non-specific lipid transfer protein from Arabidopsis is a cell wall protein. Plant $J$ $3: 427-436$

Tordesillas L et al (2013) Transport of P Pu p 3 across gastrointestinal epithelium-an essential step towards the induction of food allergy? Clin Exp Allergy 43:1374-1383

Trott 0 . Olson AJ (2010) AutoDock Vina: improving the speed and accuracy of docking with a new scoring function, efficient optimization. and multithreading. J Comput Chem 31:455-461

Wen-Zhe L (2004) Secretory Structures and Their Relationship to Accumulation of Camptothecin in Camptotheca acuminata (Nyssaceae). Acta Botanica Sinica 46:1242

Wink $M$ (2003) Evolution of secondary metabolites from an ecological and molecular phylogenetic perspective. Phytochemistry $64: 3-19$

Wright CM. van der Merwe M. DeBrot AH. Biornsti MA (2015) DNA topoisomerase I domain interactions impact enzyme activity and sensitivity to camptothecin. J Biol Chem 290:12068-12078

Zachowski A, Guerbette F, Grosbois M, Jolliot-Croquin A. Kader JC (1998) Characterisation of acyl binding by a plant lipid-transfer protein. Eur J Biochem 257:443-448

Zhao C, Li C. Wang L. Zu Y, Yang L (2010) Deter mination of camptothecin and 10-hydroxycamptothecin in camptotheca acuminata by lc-esi-ms $/ m s$. Anal Lett 43:2681-2693

Zoette V, Cuendet MA, Grossdidier A, Michielin O (2011) SwissParam, a fast force field generation tool for small organic molecules. J Comput Chem 32:2359-2368 